\title{
Characterization of the ruminal microbiota of cows raised in Brachiaria spp
}

Bruna Parapinski Santos[a]", José Antonio Bessegatto[a], Júlio Augusto Naylor Lisbôa ${ }^{[a]}$, Lais Resende Paulino ${ }^{[a]}$, Amauri Alcindo Alfieri[ ${ }^{[b]}$, Carlos Henrique Montemor ${ }^{[a]}$, Marcio Carvalho Costa ${ }^{[b]}$

[a] Department of Clinical Studies, Universidade Estadual de Londrina (UEL), Londrina, PR, Brazil

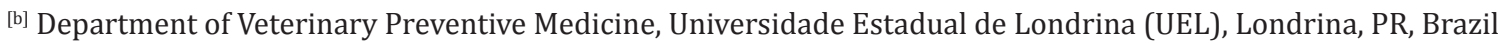

*Corresponding author

e-mail: bparapinski@gmail.com

\section{Abstract}

The ruminal microbiota is essential for life in ruminants, since it is responsible for the fermentation and production of energy, vitamins, and other essential components. The ruminal microbiota is a complex community composed by archea, bacteria, virus, fungus and protozoa. Bacteria are the main microorganism in this environment and have been studied with different methodologies, since anaerobic culturing was developed. The advent of culture independent technologies (e.g.: DNA sequencing) have changed our understanding about these communities. The objective of this study was to use high throughput sequencing to characterize the ruminal bacteria of cows raised on Brachiaria spp. pasture. Twenty Nellore cows had their ruminal fluid collected in the medial portion of the rumen right after slaughtering. DNA was extracted and the V4 region of the $16 \mathrm{~S}$ rRNA gene was sequenced using an Illumina MiSeq platform. Sequences were classified into 23 different phyla, of which, only nine accounted for more than $1 \%$ of sequences. The dominant phyla observed in all samples was Firmicutes (median 61.49\%, minimum 44.76- maximum 74.41), followed by Bacteriodes (7.13, 4.89 - 10.66), Verrucomicrobia (4.42\%, 2.26 - 8.77), Fibrobacteres (2.97, 1.15 - 17.24), Proteobacteria (2.60, 1.57 - 4.24). 529 different genera were identified, with sequences classified as Clostridiales being the most abundant (17.22\%, 8.09 - 21.34), followed by Saccharofermentans (7.94\%, 3.74-11.84) and Ruminococcus (7.42\%, 4.04 - 10.16). Results demonstrated a rich and diverse ruminal microbiota in cows kept on a pasture only diet. Great richness has been observed in the ruminal microbiota of grazing animals, which is probably related to the digestion of complex structures of fibre that requires symbiotic work of various species. Also, the ingestion of carbohydrates may decrease diversity due to rapid fermentation and consequent decrease of $\mathrm{pH}$. Imbalances in this bacterial ratio can bring adverse effects on the physiology and development, and predispose to disease. Contact with the ground, can have 
some influences due to the direct ingestion of bacteria. In conclusion, grazing cows present a rich and diverse ruminal microbiota. Firmicutes was the most abundant phylum in all animals, demonstrating the importance of these group of bacteria on the ruminal microbial ecology. Those results add to the current knowledge on the symbiotic relationship between ruminants and their bacteria and can help to understand how interferences caused by management and feeding can affect animal performance and microbial populations. 